\title{
Ambient Displays: Influencing Movement Patterns
}

\author{
Tasos Varoudis \\ 'arch+ech` architecture + technology, \\ Lab 24, Cremer Business Centre, E2 8HD, London, UK \\ varoudis@archtech.gr
}

\begin{abstract}
Ambient displays are gradually augmenting the principal static elements of architecture, such as walls, transforming space into a dynamic and ever-changing environment. Does the addition of such digital elements influence people's perception and understanding of space around them? If so, do ambient displays lead to behavioral changes like people's movement in such environments? In this particular study, a series of experiments were conducted to investigate public interior spaces with embedded ambient displays. The findings are then presented showing how the presence of an ambient display through its visual depth affects and changes movement patterns. This study discusses the ability of an ambient display to refine navigation paths and suggests that its visual depth can enhance its effectiveness.
\end{abstract}

Keywords: Ambient displays, human navigation, built environment, visual perception.

\section{Introduction}

Digital technologies have now become a medium for transforming the principle elements of architecture into dynamic components that affect people's perception and understanding of the space around them. Ambient displays, in most cases, can simply augment walls and thus have an advantage of not changing the physical morphology of space. Through light and sound, they make use of the entire physical environment as an interface and source to digital information [1]. This interface can be considered as a virtual opening, in a form of a digital window that extends the architectural space and widens the vision of users in a given space. Like a window that connects two physical spaces and transfers information between them, an ambient display can be seen as a link between physical and virtual worlds, where the virtual world can be for example the projection of another physical world or a computer generated space.

The introduction of a virtual space into the physical environment redefines the spatial properties of this environment. For instance, an ambient display can be used to transform a static wall to a more transparent and fluid element that dissolves the boundaries between virtual and physical space. During 'out of bounds' interactive installation, Chris O' Shea [2] effectively challenged the relation between physical and virtual space. By projecting previously captured spaces onto the wall, he enabled people to 'see' through the physical boundaries of space. As he stated, this interaction allowed people to enter the 'prohibited' areas of the museum while encouraging their 
childlike curiosity [2]. But how can ambient displays affect people's movement in space? The ability of ambient displays to project views of one space from another and create hybrid information space might alter our perception of space. If so, can the use of hybrid space create behavioral changes related to people's navigation in space?

The aim of this research was to analyze how digital information when introduced through ambient displays affects people's movement in the interior of public architectural spaces. The research's interest is in how the addition of an ambient display at a given space can alter people's navigation in it. In order to answer this, a series of experiments were designed to explore the effects of ambient displays on people's movement inside public spaces.

\section{Background}

Research from two fields is relevant to the work. First, this research examines work investigating the use of ambient technology in architectural spaces. Second, there is a brief outline of research studying perception and movement in architectural spaces, where ambient displays are not considered as elements that augment vision.

\subsection{Ambient Displays: Transforming Architectural Spaces}

The paradigm of ubiquitous computing represents the technological tendency of embedding information processing into everyday objects and activities. Drawing on Weiser's proposal of 'ubiquitous computing' [3], Ishii and Ulmer [4] envisioned the transformation of architectural surfaces into active interfaces between physical and virtual worlds. One of the first attempts to place an ambient display inside architectural space was initiated by Ishii et al. [5]. They designed the ambientROOM, a room equipped with ambient media (ambient light, shadow, sound, airflow, water flow). AmbientROOM provided the inhabitance of a room with a subtle awareness of human activity around people. The environment created by the ambientROOM was designed to enhance the sense of community through shared awareness concluding in a more pleasant space.

Currently, ambient displays have received considerable attention by architects who try to construct buildings with embedded digital technologies. For example, the Porsche Museum by Delugan Meissl [6] (see Figure 1) and the BMW Museum in Munich, Germany that was designed in 2007 by ART+COM [7] include large ambient displays that have augmented the interior surfaces of walls. In addition to this, when they designed Digital Pavilion in 2006, Kas Oosterhuis and Ilona Lénárd [8] transformed concrete interior walls into interfaces able to display readable information or create atmospheric lighting effects. Taking the idea of placing ambient displays instead of walls further, Ataman et al. [9] proposed the use of large display 'materials' as construction surfaces in architectural design. They envisioned movement through space in the future to be more dynamic, incorporating different levels of transparency and space that could be described by the fluidity of the walls that surround people. 


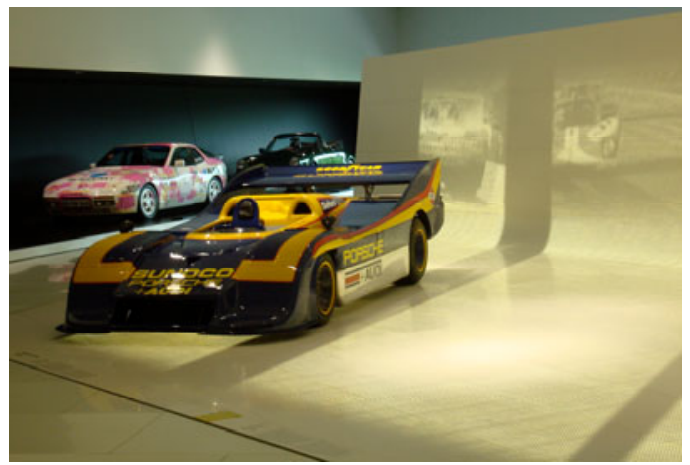

Fig. 1. Porsche Museum, Stuttgart, Germany

As indicated by previous studies [10, 11], the presence of an ambient display in architectural space can ensure more pleasant environments; while at the same time can be informative and socially engaging. Moreover, because of being blended with physical space, ambient displays are unobtrusive and do not distract the users that are not interested in the displayed content. For example, Mathew and Taylor [10] with their project AuralScapes try, with the use of sound and sky projections, to create a virtual connection from the outside to the inside, where information is abstracted and delivered at the periphery of human attention. By developing this link, they create a pleasant indoor environment and partially dissolved the static notion of the surrounding walls.

In fact, the use of digital technologies in architecture goes beyond the simple modification and transformation of space into a pleasant and informative environment, influencing people's behavior in it. With his work Röcker et al. [12] states that ambient display installations that promote awareness and presence produce positive effects and behavioral changes on office teams.

It has been observed that changes to the digital environment have led to behavioral changes such as the movement of people through that space. Indeed, Schieck et al. [13] in an attempt to analyze the influence of an interactive floor installation in people's social engagement in urban environments, reports that she recorded unexpected and diverse changes in movement patterns around the installation. But what are the changes in movement patterns resulting from the presence of ambient technology?

For architects wanting to incorporate ambient interfaces it is essential to acknowledge the effects of their design proposals on people's navigation in space. With analysis and visualization of these effects, ambient display components will be incorporated in designs efficiently and activate or transform existing spaces. Relevant studies $[6,14,15,16]$ on ambient technology place the center of attention on humancomputer interaction without considering the visual and spatial perception that link people and technology. 


\subsection{Perception and Movement in Architectural Spaces}

It is generally accepted in architecture that the structure and configuration of space affect people's navigation and movement. Gibson's research, which was primarily developed for visual perception, suggests that our senses provide us with direct awareness of the external world and its properties [17]. People perceive space through their senses and act accordingly, thus there is a tight relation between perception and movement.

Based on this theory, architect and virtual reality pioneer Benedikt [18] proposed that space is perceived as a collection of visible surfaces that are not obstructed by physical boundaries and he defined 'isovists' to describe the area in the environment that is directly visible from a location within space. A single isovist is the area of space directly visible from a given location in space, together with the location of that point. For example, in a convex space or a rectangular space with partitions the isovist area of a given point may not include the full area of that space and some parts of the space will not be directly visible from other points in space (see Figure 2).

Another urban and architectural theory that is relevant to this study is 'Space Syntax'. Hillier and Hanson proposed 'Space Syntax' to describe and analyze the character of a space and its effects on human behavior [19]. 'Space Syntax' research shows that the majority of human movement occurs along the longest lines of sight, and that the more open visible space we have in front of us the more we tend to move towards that direction [20]. However, the complexity of the spatial elements that are taken into consideration is limited. 'Space Syntax' sees space as a set of solid walls and empty openings and does not examine transparent elements. In addition to the lack of consideration of transparent materials, there is also a lack in understanding the effects of ambient technologies and 'digital' transparencies. Both physical and 'digital' transparencies may have important effects on people's perception of space and movement within it as they both extend and sometimes distort the depth of field.

Existing spatial analysis theories do not take into consideration complex architectural components such as ambient displays. Despite the fact that there are

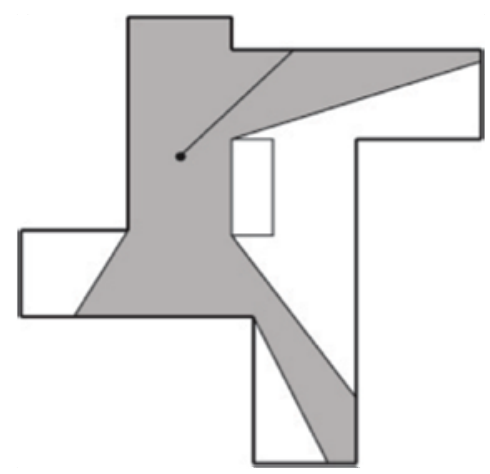

Fig. 2. Isovist field: grey color indicates the visible area from point 
some studies that deal with pervasive systems in urban environments, they focus on social behavior and do not consider the influence of ambient displays on human movement [13, 21]. Additionally, such research is limited in considering ambient technology as a layer that is placed over existing urban infrastructures or simply replaces building facades. As we move into a world where a fusion of virtual and physical is going to be prevalent [22], studying and analyzing people's behavior in relation to the use of embedded ambient displays can offer important knowledge.

\section{Aims and Objectives}

The study starts with the assumption that the topological and visual relations between physical spaces are two important factors that determine the distribution of people's movement in space [23].

This research intends to analyze the changes in movement patterns when ambient displays are used as a virtual extension of the visual boundaries inside public architectural spaces. The hypothesis of this study is that placing ambient displays that virtually link and extend one physical space towards another that is near but not directly accessible will influence the topological and visual relations between spaces and as a result will affect the distribution of people's movement (see Figure 3).

Analyzing the effects of ambient displays on movement patterns when are blended in architectural spaces and induce virtual augmentation would provide a better understanding of the relation among humans, space and ambient displays. In contrast to current stable physical spaces, the introduction of new responsive and everchanging materials will impose new fully adaptive architecture. Therefore, extending current knowledge and theories to involve digital transparencies as a crucial element of spatial configurations is inevitable.

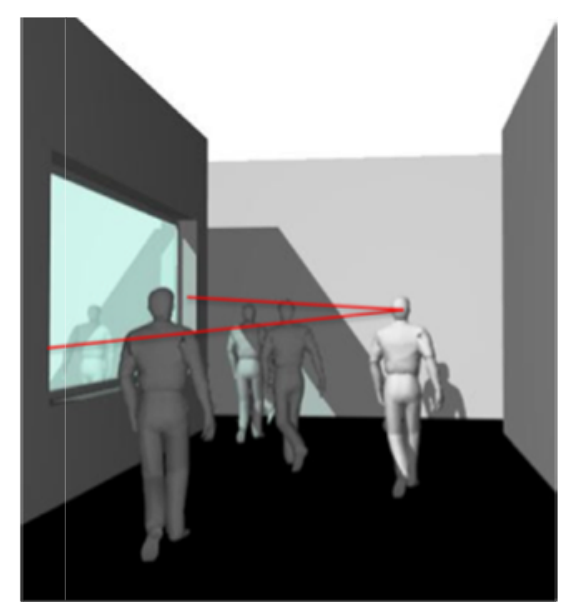

Fig. 3. Ambient display as digital transparency 


\section{Methodology}

To achieve the aims of this research, a series of experiments were developed in order to observe and analyze people's movement in different spaces depending on the presence of ambient displays (see Figure 4). The experiments are focused on the use of ambient displays in the interior of public architectural spaces by placing them near a place of common interest. The main reason behind the use of public architectural spaces is that public spaces offer great opportunities for experimenting with ambient technology and the analysis of movement. From a methodological perspective, such places allow observing the movement behavior of a significant amount of people providing a larger sample for experimentation and analysis.

A fundamental form of space, in which it is simple to examine the flow and direction of people between discrete routes, is corridor-like settings. Based in this setting, two distinguished routes are needed, from which the users can choose in order to access the target space. 'Target space' is considered as a space with common interest for the participants of the experiment such as a coffee area or a common room that they can prepare and eat their lunch.

This type of experiment gives the opportunity to examine a single 'decision making' point providing clear and unbiased experimental conditions. It is essential that the routes need to be symmetrical in order not to influence the users' choice by producing different visual triggers. Moreover, having the ' $\mathrm{T}$ ' shaped corridors as the setting, where people can go left or right, gives symmetrical visual characteristics. Therefore, the addition of an ambient display just before the corner as depicted in Figure 5 is expected to act as a visual trigger. The display's position near the corner will potentially introduce a digital opening in the visual field and will influence people's movement.

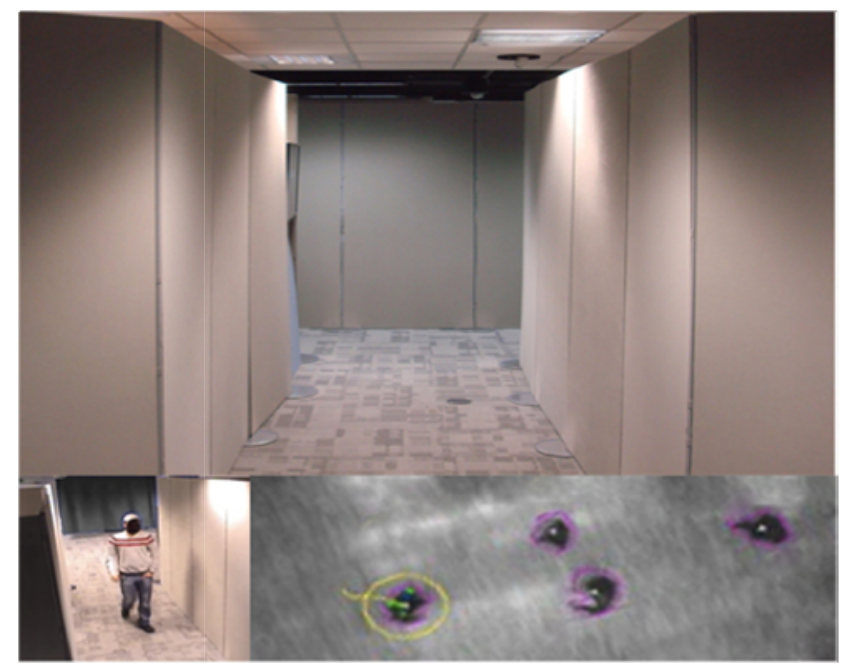

Fig. 4. Example of an experiment setting. Ambient display embedded in wall to the left. 

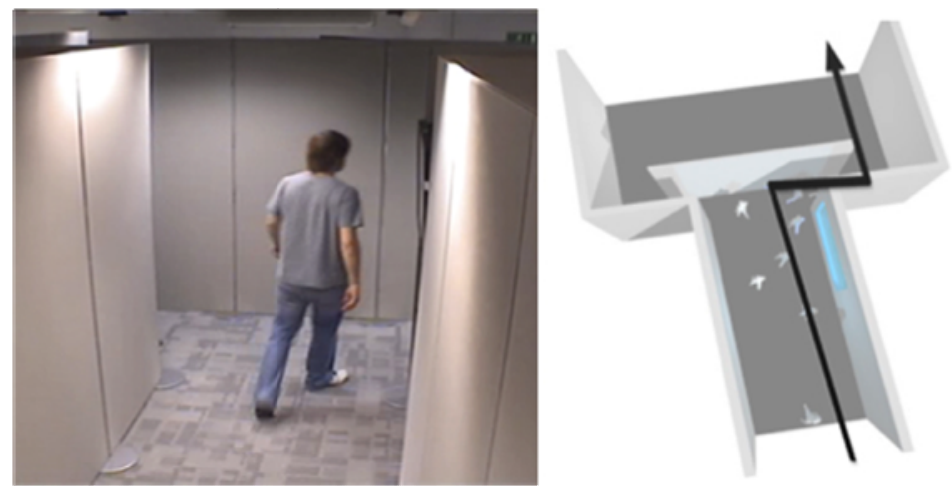

Fig. 5. Influencing direction of movement. Ambient display embedded in wall to the right.

Intuition and a simplistic interpretation of 'Space Syntax' suggest that the presence of an ambient display in a corridor should not influence the route decision-making choices of occupants going down this corridor. In the condition when both routes are equidistant from the objective, one might well expect a 50/50 left-right split of occupants. Alternatively, the hybrid space hypothesis suggests that a digital ambient display that functions like a virtual window will alter spatial morphology and so will result in an observable behavioral change in movement patterns (see Figure 5). To test this, a set of corridors as a 'T-shaped' arrangement was constructed with the upper part of the ' $\mathrm{T}$ ' near the target space. As the 'target space', a room for coffee providence and preparation was used, since it is more attractive for daily visits for both staff and visitors. The research's interest is to examine how the addition of an ambient display would affect this distribution, a case that currently is not predictable by relevant theories like 'Space Syntax'.

Having set an outline of the experimental requirements, the design phase divided in three parts. The first part is focused on the ambient display and its content while the second part on spatial setting. The third part is a combined effort for the effective placement of the ambient display in the particular space.

Deriving from the background research and the hypothesis, the display's main function was to act as a live video link providing a one-way video from the 'target space' and introducing an augmented opening that digitally expands vision. In addition, a series of 'null-tests' were introduced as part of the experiment using the ambient display with: no content, random static images and random animated content. The 'null-tests' were critical for establishing the content of the display as the only source of influence.

\subsection{Experimental Set-Up}

The space used was the Ambient Technology Lab at the Open University UK. It is a space of approximately $144 \mathrm{~m} 2$ that is free of internal walls and can be configured in 
any arrangement easily with little limitations. For the construction of the corridor-like setting lightweight solid partitions were used covering the full height of the space and positioned in order to produce a symmetrical space. The remaining space at the end of the corridor-like setting was designed as a small cafeteria with sufficient space for people to stay and chat with each other reproducing a casual everyday atmosphere. Over the course of the experiment there was free coffee, tea and some sweets in this space available to everyone.

For the data gathering, a multi-camera set-up was used for synchronized recording of every corner of the lab. The cameras were positioned in the ceiling and configured to track multiple angles of the decision-point area as well as the full length of the corridor and the coffee area. Additionally, the cameras could also focus on the face along with the body of each subject in order to recognize the line of sight, as each subject was moving towards and passing the decision point. For every day of the experiment eight video streams were recorded during the working hours.

The experiment ran over a period of several weeks where people that work in the university or visitors could use this setting for taking their morning or afternoon coffee. None of the participants were aware of the research's nature but were informed that they were taking part in an experiment and would be recorded. The experiment area was clearly marked to the effect that they are entering a video monitored zone and emails sent out to ask for participants. The emails explained that the purpose of the experiment will be revealed after its compilations and the whole process was ethically approved. While participants were asked to use a specific entrance and exit door in order to avoid passing through the corridor in both directions, it was felt that this did not bias participants' response of direction choice.

As discussed before, the measurements had to be compared with a 'base model' of this space with no ambient displays installed. On that account, the experiment started by collecting data about people's movement within the T-shape configuration with no ambient display present. After having sufficient data to serve as the basis of the experiment, the second phase started, in which a large display was carefully embedded into the right wall (see Figure 6) of the constructed corridor just before the corner (decision point). The display, a large anti-glare display with a $15 \mathrm{~mm}$ black casing, was linked with a high-definition professional camera and depicted the coffee area as seen from a particular 'perspective'. This 'perspective' view was used in order to emulate the actual perspective of the coffee area that an opening at this position would have revealed. Similarly, in the third phase the display was placed symmetrically on the left side of the corridor, positioned in the same way and producing the same effect but streaming data of the coffee area as seen from the left side. Everything else in the experimental setting remained unchanged for the course of the experiment. At the end of the study all video streams were examined regarding the movement, choice of direction and the reactions of the people. All data were treated with confidentiality and not shared with anyone outside the limits of this research. 


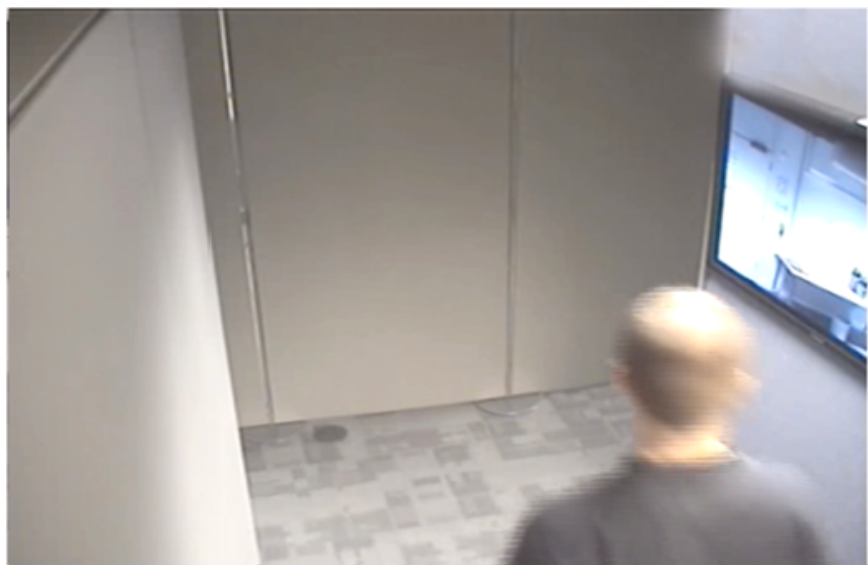

Fig. 6. Example of an experiment setting. Ambient display embedded in wall to the right.

\subsection{Initial Reactions - Video Observation}

To capture people's initial reactions to the ambient display the recorded video, which was taken from cameras tracking people's movement while approaching the decision point, was observed. Upon approaching the display a small percentage of people were seen to momentarily stop and look at it for just a second and then turn towards the route of the screen. The majority of people only seemed to take a glimpse of the screen by turning their head slightly and then turn at the corner. Although the video revealed that most of the individuals took their decision quickly, some individuals changed their initial decision and finally moved towards the side of the display. Overall, while the ambient display was something new to their environment less than $0.01 \%$ of people who took part in the experiment seemed unfamiliar to its presence. In total, more than 800 individuals took part in the experiment, which gives us a clear evaluation of the hypothesis. The number of participants doesn't include people passing multiple times but the observations are kept in order to later check their potential contribution on the overall hypothesis.

\section{Results}

To analyze the movement patterns in the experimental setting the number of individuals and groups walking through the corridor were counted in relation to their decision to follow the right or left direction in the particular setting. The data were categorized according to the experimental phase (without display, with the display on the left and with the display on the right) and whether it was individuals or groups. The analysis conducted by grouping the data into two categories in order to eliminate any signs of internal influence within the groups of people ('groups' have more than one person and all subjects follow the first person in the group): 1) for 'all groups and individuals' without taking into account if a person was alone or a part of the group and 2) 'only individuals' counting all 'individuals' and each of the 'groups' as one 
subject unaffected by the number of people in the group. These categories were analyzed using a chi-squared test and logistic regression analysis (modeling of binomial proportions).

In the first phase (no display), which took place over a period of four weeks, the findings revealed that combining groups and individuals, $55 \%$ of the people turned left in the specific setting and $45 \%$ turned right, while counting only the individuals the distribution was $54.8 \%$ left and $45.2 \%$ right (see Figure 7).

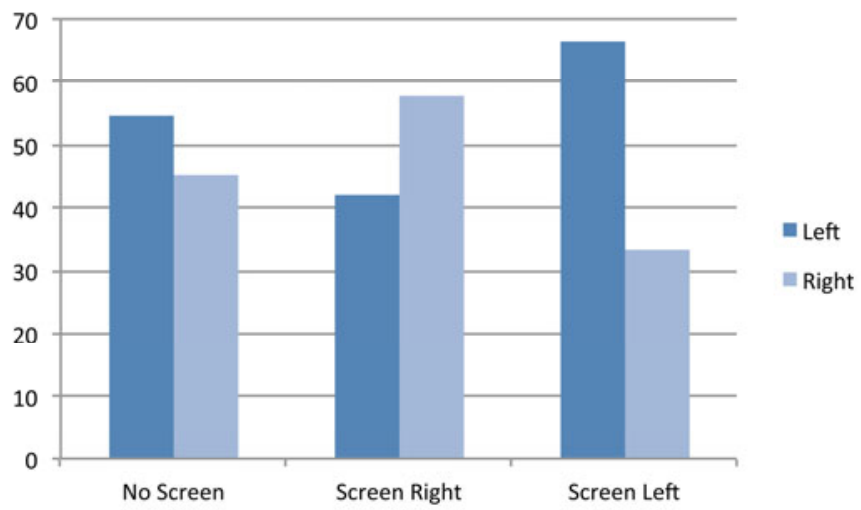

Fig. 7. Distribution $(\%)$ between routes

In the second phase where the display was on the right side and run over a period of three weeks, the distribution was $41.1 \%$ turning left and $58.9 \%$ turning right for the combined test subjects and $42.1 \%$ left and $57.9 \%$ right for only the individuals (see Figure 7). Those results showed a significant 'shift' in people's distribution along the two alternative directions. The shift towards the right side was $13.9 \%$ combining all test subjects together and $12.7 \%$ counting only the individuals.

Finally, in the third phase with the display positioned on the left side and run for three weeks, the distribution was $73.4 \%$ turning left and $26.6 \%$ turning right for the combined test subjects and $66.6 \%$ left and $33.4 \%$ right for only the individuals. Those results also showed a significant 'shift' in people's distribution (see Figure 7). The shift towards the left side was $18.4 \%$ combining all test subjects together and $11.8 \%$ when counting only the individuals.

Furthermore, for validation of the significance of the results from the three unmatched groups a chi-square statistical test was used. The p-value of this test was 0.000047. Figure 8 depicts the calculated 'expected' percentages from the chi-square test against the observed percentages.

Following the tests about the general significance of the measured data and the influence of the ambient display, a logistic regression analysis was conducted ('modeling of binomial proportions'). For this test a value denoting the position of the ambient display was introduced (direction of influence): ' -1 ' when the ambient display is at the opposite side than the one testing, ' 0 ' when there is no display and ' 1 ' when the display is on the same side, as well as a value denoting the presence of an ambient display regardless of position (encoded as ' 0 ' for not present and ' 1 ' for 
present in one of the walls). The 'direction of influence' matches the direction of the significant change in movement as measured in the experiment, with a p-value (t pr.) less than 0.001. The result of this analysis further confirms the hypothesis that the ambient display produces a significant change in the direction of movement towards the side at which the display is located. Additionally, combining the direction of influence' and the 'presence' variables evinces that the resulted distortion is not based on the side of the corridor, where the ambient display is located, and thus for both 'right' and 'left' cases the change was equally significant.

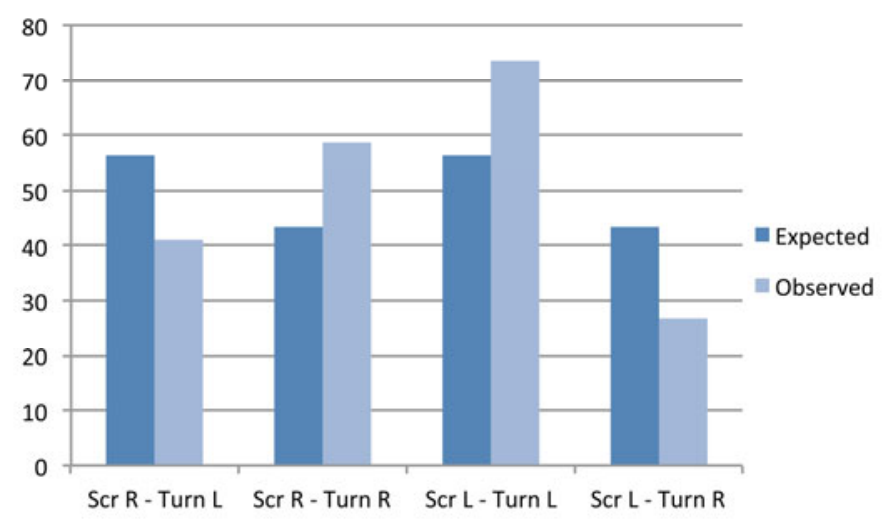

Fig. 8. Expected and observed (\%) movement distribution

\subsection{Null-Tests Results}

The results presented until now showed that the presence of an ambient display influences how people move through the environment. However there was a need for extracting whether the ambient display itself (the device) or its content were responsible for that change. For that reason, using the same equipment and spatial setting a follow-up experiment was conducted positioning the display in the same places as the first experiments but without the video link. Instead of the video feed, several scenarios were tested: no image is used in order to test the ambient display as a device while static images, animated images and short video clips used to explore the effects of diverse content that could affect the users.

The results were treated in the same way as the first experiment and showed that the shift in direction between the phase without a display (base model) and each of the null-test phases is less than $3 \%$ in all cases and not statistically significant.

In detail, from more than 400 individuals who took part in the null-test experiments, the majority of people continue to use the left route more (the same way as before the application of the ambient display), with the percentage varying from $57 \%$ to $53 \%$ in favor of the left route. More analytically, compared to the phase without an ambient display where the distribution was $55 \%$ left and $45 \%$ right, the distribution with the display on the left for the worst case null-test scenario (case with the largest shift) was, $57.8 \%$ left and $42.2 \%$ right; while with the display on the right, 
$53.2 \%$ left and $46.8 \%$ right. What is clearly visible from the results is that the ambient display blends in the environment and becomes an unobtrusive object that without the visual extension does not influence people's behavior and movement in space.

\section{Conclusion}

The results of this study reveal that, an ambient display that shows typical information has, as one might expect, no change on pedestrian route choice behavior. If the display shows a projection of nearby space, relevant to the pedestrian, then the presence of the augmented visual depth and more particularly the position of the ambient display influences route choice behavior. This has a number of ramifications in terms of both the design of ambient displays in architectural settings and the use of augmented/hybrid spaces in research conditions.

Combining the findings from the experiment and the observation from the recorded video it is concluded that there is a significant change in the distribution of people and their movement patterns when an existing architectural space is augmented by the presence of an ambient display projecting a virtual link between two physically disconnected spaces (where the one can be also a virtual representation of a space). It is also clear from the results that the position of the display in the symmetrical routes has no different effects regarding the distortion of movement. In addition to this, the results of the 'null-tests' addressed that when the ambient display is not producing an augmentation of the visual field or the visual depth, it does not influence people's movement in space.

This study also demonstrates that the position of the ambient display as a visual link to a near but physically disconnected location has the effect of increasing awareness about this space giving a subliminal direction towards the side where the screen is situated. In some cases the ambient display unconsciously nudged people to pass through the corridor setting and go to the coffee area. As the video revealed, most of the users took decisions quickly. However, there were cases of individuals changing their initial decision and adapting their route towards the side of the screen. It is speculated that raising awareness of this decision may have increased the likelihood that these individuals recalculated the new augmented layout of space against the old and moved accordingly.

Accepting that people's visual perception of space becomes influenced by the virtual augmentation and extension of space that ambient displays produce, this research can contribute in two main areas. One area deals with the architectural space design featuring ambient displays, as well as navigation in space. The second area is the content and interface design for ambient media. In the fast-growing field of digital augmentation in architecture, understanding and acknowledging people's movement, proximity and navigation in space can give new ways of managing and directing movement towards desired places or interfaces. Examples within this area include subliminal nudges for accessibility of remote or 'hidden' spaces as well as alternative and more efficient methods to assist way-finding. In addition, ambient information systems can be made and positioned in a way able to enhance interactivity and social engagement. 
In the field of human-computer interaction the design of the interface is of crucial meaning for the effectiveness of the ambient displays. As it is clear that people react to the virtual depth of the ambient interface, as indicated by the change in movement within the experiments, designing such interfaces needs a significant consideration of the results that they will induce. As this study shows, an ambient display that extends the visual depth acts as a subliminal element attracting people to approach it and also change their direction. This effect is clear when comparing the findings from the 'null-test' with the main experiment.

Further research is needed, however, to produce more generic insights that might help with ambient display usage in architectural space and more critically the interface design itself. For this reason, it is crucial to explore more areas of the visual augmentation of spatial boundaries with ambient displays. Currently, a research that explores how the directionality of the virtual perspective projection, which is produced by ambient displays, can influence people's direction of movement and proximity from the display is in its final stages. In essence, the intention is to produce attraction and movement by using skewed perspective projections on statically positioned ambient displays.

This research suggests that embedding ambient displays that extend the visual depth into space can engage people to adhere to certain kinds of desired movement patterns.

Acknowledgments. The author would like to thank Katerina Alexiou, Sheep Dalton, Theodore Zamenopoulos and Jeff Johnson for their immense support throughout the study, Professor Paul Garthwaite for the statistical analysis and Zeta Kachri for helping, proofreading and editing the paper.

\section{References}

1. Wisneski, C., Ishii, H., Dahley, A., Gorbet, M., Brave, S., Ullmer, B., Yarin, P.: Ambient Displays: Turning Architectural Space into an Interface between People and Digital Information. In: Yuan, F., Konomi, S., Burkhardt, H.-J. (eds.) CoBuild 1998. LNCS, vol. 1370, p. 22. Springer, Heidelberg (1998)

2. O'Shea, C.: http: / /www. chrisoshea .org/projects/out-of-bounds /

3. Weiser, M.: The Computer of the 21st Century. Scientific American 265(3), 66-75 (1991)

4. Ishii, H., Ullmer, B.: Tangible Bits: Towards Seamless Interfaces between People, Bits and Atoms. In: Proc. of Conference on Human Factors in Computing Systems (CHI 1997), pp. 234-241. ACM Press, New York (1997)

5. Ishii, H., Wisneski, C., Brave, S., Dahley, A., Gorbet, M., Ullmer, B., Yarin, P.: AmbientROOM: Integrating Ambient Media with Architectural Space. In: Summary of Conference on Human Factors in Computing Systems, CHI 1998, pp. 173-174. ACM Press, New York (1998)

6. Porsche Museum (2009), http://www.porsche.com/uk/aboutporsche/porschemuseum/

7. ART+COM BMW Museum (2008), http : / /www . artcom.de/index.php? lang=en\&option=com_acprojects \&id $=64 \&$ I temid $=144 \&$ page $=6$ 
8. Oosterhuis, K., Lénárd, I.:

http: / / www. oosterhuis.nl/quickstart/index.php?id=492

9. Ataman, O., Rogers, J., Ilesanmi, A.: Redefining the Wall: Architecture, Materials and Macroelectronics. International Journal of Architectural Computing 04, 125-136 (2006)

10. Mathew, A., Taylor, J.: AuralScapes: Engaging Ludic Ambiguity in the Design of a Spatial System. In: Proc. of CHI 2008, pp. 2533-2542. ACM Press, New York (2008)

11. Tomitsch, M., Moere, A., Grechenig, T.: A Framework for Architecture as a Medium for Expression. In: Workshop on Pervasive Visual, Auditory and Alternative Modality Information Display, Pervasive (2008)

12. Röcker, C., Prante, T., Streitz, N.A., van Alphen, D.: Using Ambient Displays and Smart Artefacts to Support Community Interaction in Distributed Teams. In: Proc. of the OZCHI Conference, OZCHI 2004 (2004)

13. Fatah gen. Schieck, A., Briones, C., Mottram, C.: A Sense of Place and Pervasive Computing within the Urban Landscape. In: Proc. 6th International Space Syntax Symposium (2007)

14. Prante, T., Rocker, C., Streitz, N.A., Stenzel, R., Magerkurth, C., van Alphen, D., Plewe, D.A.: Hello. Wall- Beyond Ambient Displays. In: Dey, A.K., Schmidt, A., McCarthy, J.F. (eds.) UbiComp 2003. LNCS, vol. 2864, pp. 277-278. Springer, Heidelberg (2003)

15. Jafarinaimi, N., Forlizzi, J., Hurst, A., Zimmerman, J.: Breakaway: an Ambient Display Designed to Change Human Behavior. In: Proc. CHI 2005, pp. 1945-1948 (2005)

16. Consolvo, S., McDonald, D.W., Landay, J.A.: Theory-Driven Design Strategies for Technologies that Support Behavior Change in Everyday Life. In: Proc. CHI 2009, pp. 405-414 (2009)

17. Gibson, J.: The Ecological Approach to Visual Perception. Houghton Mifflin, Boston (1979)

18. Benedikt, M.: To Take Hold of Space: Isovist and Isovist Fields. Environment and Planning B 6, 47-65 (1979)

19. Hillier, B., Hanson, J.: The Social Logic of Space. Cambridge University Press, Cambridge (1984)

20. Hillier, B., Penn, A., Hanson, J., Grajewski, T., Xu, J.: Natural Movement: or, Configuration and Attraction in Urban Pedestrian Movement. Environment and Planning B: Planning and Design 20, 29-66 (1993)

21. Fatah gen. Schieck, A., Lopez de Vallejo, I., Penn, A.: Urban Space and Pervasive Systems. In: Proc. The Seventh International Conference on Ubiquitous Computing (2005)

22. Spiller, N.: Cyber_Reader: Critical Writings for the Digital Era. Phaidon Press, New York (2002)

23. Hillier, B.: Space is the machine. Syndicate Press, University of Cambridge, UK (2004) 